\title{
Can the hamstring muscles protect the anterior cruciate ligament during a side-cutting maneuver?
}

\author{
E. B. Simonsen, S. P. Magnusson¹, J. Bencke, H. Nasborg, M. Havkrog, J. F. Ebstrup, H. Sørensen \\ Institute of Medical Anatomy, section C, The Panum Institute, University of Copenhagen, ${ }^{1}$ Team Denmark Testcenter, Bispebjerg \\ Hospital, Copenhagen, Denmark \\ Corresponding author: Erik B. Simonsen, Ph.D., Institute of Medical Anatomy, section C, The Panum Institute, University of \\ Copenhagen, Blegdamsvej 3, DK-2200 Copenhagen N, Denmark
}

Accepted for publication 15 July 1999

\begin{abstract}
Because anterior cruciate ligament (ACL) injuries are common in European handball the present study assessed knee joint shear forces to estimate ACL loading in six elite female handball players during a side-cutting maneuver. A pilot investigation in three dimensions showed that peak moments occurred in the sagittal plane at a high velocity. Therefore, analysis of the movement was performed in two dimensions using high-speed cinematography, ground reaction forces, and electromyography (EMG). Film and force plate data allowed for calculation of net joint moments (inverse dynamics), estimates of instantaneous muscle-tendon lengths, contraction velocities, and peak loading of the ACL. During the breaking phase of the maneuver the peak
\end{abstract}

knee joint moment was $239 \mathrm{Nm}$ (99-309), which yielded an ACL-load of 520 N (215-673). The corresponding peak EMG amplitudes for the hamstring muscles were 34-39\% of maximum EMG. During the breaking phase the quadriceps muscle contracted eccentrically with a velocity of 216-253\% fiber length/s. In constrast, the hamstring muscles contracted concentrically with a velocity of 222$427 \%$ fiber length/s. These results suggest that a side-cutting maneuver produces loads that are insufficient to rupture the ACL. Furthermore, the rapid concentric hamstring contraction suggests that even during maximal activation, the ability of the hamstrings to reduce the ACL load is marginal.
Disruption of the anterior cruciate ligament (ACL) in sports is not an uncommon injury (Emerson 1993, Lindblad et al. 1992, Myklebust et al. 1994, Strand 1993). Although our understanding and both conservative and surgical management of these injuries have advanced, it remains a problem for the clinician and patient.

Most frequently the ACL injury is sustained during non-contact situations in sports that involve deceleration and/or rapid changes of direction (Strand et al. 1990). While these situations require that the knee is mobile it also demands simultaneous stability. The stability may be static and/or dynamic in nature. The bony configuration, joint capsule and ligaments provide the static restraints and the hamstring muscles may provide dynamic support (Solomonow et al. 1989, Andriacchi \& Birac 1993). Movement of a limb segment typically involves some degree of agonist and antagonist co-contraction (Draganich et al. 1989). Therefore, intuitively, deceleration or a change of direction during side-cutting, which requires considerable quadriceps activation, should be accompanied by substantial hamstring co-contraction. Such hamstring activity would theoretically serve to decrease the shear forces on the tibia and consequently minimize the load placed on the ACL (Solomonow et al. 1987). It is this notion that the hamstring muscles can reduce the load on the ACL that prompted emphasis on hamstring muscle strengthening regimens during post ACL surgery, for conservative treatment of ACL injury and in attempts to prevent ACL injury (Bencke et al. 1999). However, whether the hamstring muscles contribute significantly to reduced shear forces, and thereby ACL loading, during sports activity remains unknown.

European team handball is a team sport that has a remarkable incidence of ACL injuries, which are typically sustained during side-cutting maneuvers (Myklebust et al. 1994). Furthermore, it appears that the incidence is disproportionately high in female players (Myklebust et al. 1994, Strand 1993). Therefore, the purpose of the present study was to assess knee joint shear forces to estimate ACL loading in women during a side-cutting maneuver and to investigate the possibility of the hamstring muscle group being able to reduce loading of the ACL during this movement. 


\section{Material and methods}

Pilot study

The present study was based on pilot data of a side-cutting maneuver typical for European team handball. The methodology and the results of this study will therefore be described briefly here.

Nine female elite team handball players were studied during a side-cutting maneuver. The subjects were filmed by three video cameras (Panasonic WV-GL350) during a side-cutting maneuver typical for European team handball. The ball was passed to the subject during a two-step run-up after which the subject performed the movement pattern with one foot on a force platform (AMTI OR6-5-1). Reflective markers were placed over the following anatomical landmarks: the fifth metatarsal joint, the ankle joint, the knee joint, the hip joint and the top of the iliac crest. Three-dimensional coordinates were derived by a video analysis system (Peak Performance Inc., Colorado, USA) and net joint moments were calculated by inverse dynamics with the use of $\mathrm{x}$ - and $\mathrm{y}$-coordinates combined with the force plate recordings. The calculations showed that the peak moment about the knee joint occurred exclusively in the sagittal plane, i.e. the movement pattern consisted of a decelerating part performed in the sagittal plane, while the accelerating part, the take-off, was performed partly in the transversal plane. Similar observations have been reported by Colby et al. (1997). In addition, instantaneous origin to insertion lengths were calculated for the most important muscle-tendon units, including the hamstrings. These calculations showed that the hamstring muscles were shortening rapidly during the braking phase when the quadriceps muscle dominated the knee joint moment. Therefore, because peak moments occurred strictly in the sagittal plane at a relatively high velocity of movement it was decided to further analyze the movement in two dimensions using high-speed cinematography and electromyography (EMG). The remaining portion of the study strictly addresses the two-dimensional part recorded on high-speed film.

\section{Subjects}

Six female subjects volunteered to participate in the study, which was approved by the local ethics committee. All subjects were elite players from the highest division of team handball in Denmark. The mean age was 21 years (18-23), body weight 66 $\mathrm{kg}(56-78)$ and height $1.69 \mathrm{~m}$ (1.64-1.73).

\section{The side-cutting maneuver}

The purpose of the side-cutting maneuver is to pass by a defending player by faking the direction opposite to the intended movement. Normally, a right-handed shooter will approach the defending player head on, touch down on the left foot, brake the forward velocity, and step to the right side (intended direction). During the braking action, the $\mathrm{m}$. quadriceps femoris contracts eccentrically causing an anteriorly directed shear force on the tibia that stresses the ACL (Colby et al. 1997). The movement pattern is similar to that performed in basketball and American football as well as European team handball.

\section{Experimental procedure}

After a short warm-up period, when the subjects were allowed to get accustomed to the laboratory conditions, maximal voluntary EMG amplitudes were measured during isometric conditions. Three trials were recorded for each muscle group and the subjects were verbally encouraged to perform maximally. Then the subjects performed 10 consecutive trials of the sidecutting movement during which only EMG and ground reac- tion forces were measured. Finally, three trials were recorded on high-speed film synchronized to EMG and ground reaction forces.

\section{Cinematography}

The movements were filmed at $200 \mathrm{~Hz}$ with a high-speed 16$\mathrm{mm}$ camera (Teledyne DBM45) placed $8 \mathrm{~m}$ from the force platform facing the subject in profile. The direction of movement was always from right to left and only the left leg was used for the analysis (all subjects were right-handed shooters). Reflective markers were glued over bony protuberances corresponding to the lateral top of the iliac crest, the greater trochanter, the femoral epicondyle, the lateral maleolus and the fifth metatarsal joint. In addition, the borders of the force platform were marked. A square wave signal generated by the camera indicated the exposure of each frame, and this signal was sampled together with the other analog signals to verify that the frame rate was correct and that the camera had reached constant speed at the time of synchronization. The latter was accomplished by lighting a small diode in the field of view and simultaneously sending a trigger signal to an AD-converter (DT2801-A) installed in a personal computer. All analog signals were sampled at $1 \mathrm{kHz}$. The $16 \mathrm{~mm}$ film was developed and transferred to video tape using a highly accurate device specially designed for this purpose (ELMO TRV-16GICCD) that projects the film at 25 frames per second while it is also being filmed at 25 frames per second by a built-in video camera. This process means that the even and odd video fields forming one video frame are identical. Accordingly, only the even fields of the videotape were digitized and the geometric joint centers of the joint markers were traced by an automatic software module (Peak Performance Inc., Colorado, USA).

\section{Ground reaction forces}

The force platform (AMTI OR6-5-1) measured $0.5 \times 0.4 \mathrm{~m}$. The resonance frequency was about $500 \mathrm{~Hz}$ in all directions. The vertical force (Fy), the sagittal force $(\mathrm{Fx})$ and the moment of torsion $(\mathrm{Mz})$ about the transversal axis were measured. The Fy and the $\mathrm{Mz}$ were used to compute the center of pressure (COP)

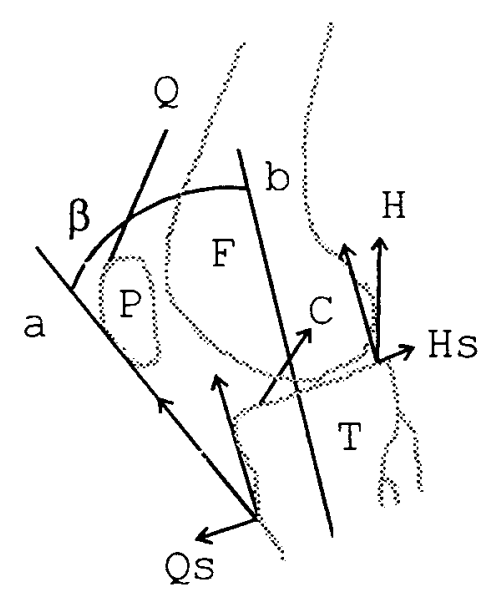

Fig. 1. The model used to calculate shear forces and thereby ACL-loading. The angle $\beta$ between $a$ and $b$ represents the orientation of the patellar ligament, which varies with the knee joint angle. Qs: shear force component from $\mathrm{m}$. quadriceps femoris. $\mathrm{C}$ : ACL force. Hs: shear force component from the hamstring muscles. Q: muscle force from $\mathrm{m}$. quadriceps femoris. $\mathrm{H}$ : muscle force from the hamstring muscles. F: femur, T: tibia. P: patella. 


\section{Simonsen et al.}

on the force platform and, based on the location of the platform with respect to the film recordings, the COP was aligned to the ground foot.

\section{Electromyography}

Surface electrodes (Medicotest Q-10-A prefilled ECG electrodes) were mounted $2 \mathrm{~cm}$ apart over the most prominent part of the muscle belly on the following muscles: $\mathrm{m}$. tibialis anterior (TA), m. soleus (SO), m. gastrocnemius lateralis (GAL), m. gastrocnemius medialis (GAM), m. vastus medialis (VM), m. vastus lateralis (VL), m. semimembranosus and semitendinosus (SMT), m. biceps femoris caput longum $(\mathrm{BFcl})$ and $\mathrm{m}$. gluteus maximus (GM). The skin was shaved, wrapped with emory cloth and cleaned with pure alcohol prior to mounting the electrodes, which were connected to small custom-built pre-amplifiers taped to the skin. The pre-amplifiers were 1:1 followers (input impedance $80 \mathrm{M} \Omega$ ), which only served to lower the impedance to reduce movement artifacts. From the pre-amplifiers the signals were led through 10-m-long shielded wires to custom-built amplifiers with a frequency response of $20 \mathrm{~Hz}$ to 10 $\mathrm{kHz}$. The wire setup permitted the subjects to move freely. Nei- ther tapping the leg nor passive movements produced any visible artifacts.

\section{Signal treatment}

The EMG signals were digitally highpass filtered at $20 \mathrm{~Hz}$, full wave rectified and then lowpass filtered at $15 \mathrm{~Hz}$ to produce linear envelopes (Butterworth fourth order zero lag digital filters).

After the calculation of COP on the force platform the sampling frequency of the force signals was reduced by averaging five data points to match the $200 \mathrm{~Hz}$ frame frequency of the film. The averaging of five data points ensured that Fx, Fy and COP values corresponded to each frame of the film.

The time-position data of the joint markers were digitally lowpass filtered at $5 \mathrm{~Hz}$ (Butterworth fourth order zero lag lowpass filter) to minimize measurement inaccuracies from film digitization. Spectral analysis (FFT) assured that less than $10 \%$ of the total spectral energy was removed by the lowpass filter. An "optimal cutoff frequency" was determined for each marker by the "Jackson Knee" method (Jackson 1979) and the method based on residual analysis proposed by Winter (Winter 1990). However, both methods resulted in noisy joint moments accumulating
Fig. 2. Data from a typical trial of one subject. At the top, stick-diagram of the side-cutting maneuver, every 10th frame of the film taken at $200 \mathrm{~Hz}$ is displayed. The vertical (Fy) and sagittal $(\mathrm{Fx})$ ground reaction forces. The net knee joint moment (positive values indicate extensor dominance). Linear envelope EMGs (shaded) of $\mathrm{m}$. vastus medialis $(\mathrm{VM}), \mathrm{m}$. vastus lateralis $(\mathrm{VL}), \mathrm{m}$. gluteus maximus (Gmax), m.m. semimembranosus et semitendinosus (SMT) and $\mathrm{m}$. biceps femoris caput longum $(\mathrm{BFcl})$. The EMGs are expressed relative to the maximal EMG measured during isometric conditions. Superimposed on the EMGs are instantaneous muscle-tendon lengths, each scaled from minimum to maximum. Inflection indicates lengthening while deflection indicates shortening. Note that the biarticular hamstring muscles contract concentrically during the braking phase. Vertical dotted lines indicate from left to right: touch down, end of braking phase and toe off.

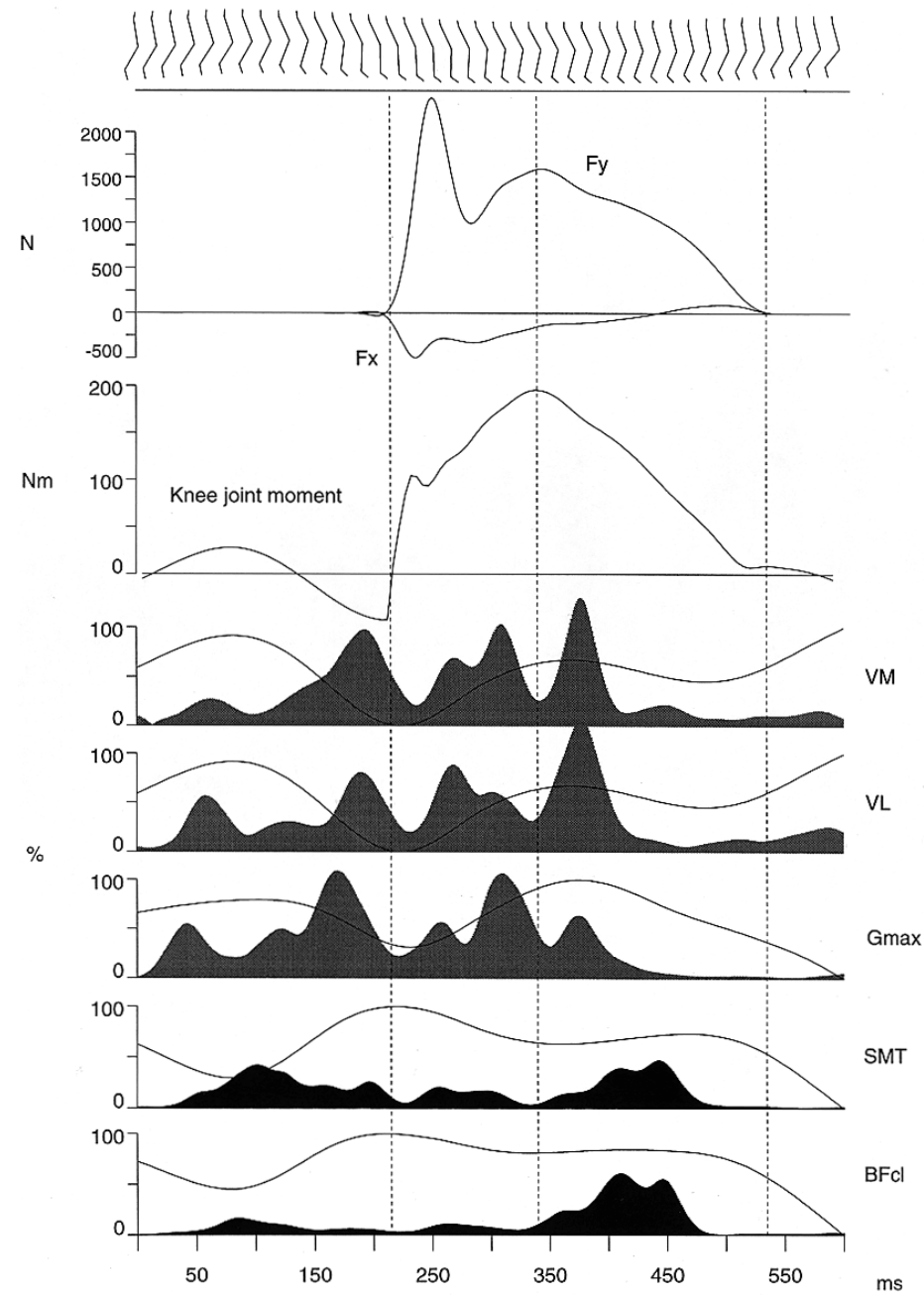


from the ankle joint to the hip joint obviously caused by too high cutoff frequencies. Based on the spectral analysis, it was therefore decided to filter all coordinates at $5 \mathrm{~Hz}$.

\section{Dynamic calculations}

The $\mathrm{x}$ - and $\mathrm{y}$-coordinates of the joint markers were used to form a link-segment model consisting of four segments: pelvis, thigh, shank and foot. The filtered time-position data were used to compute linear velocities and accelerations as well as joint angular velocities and accelerations.

By the use of film and force plate data, net joint moments were calculated about the ankle, knee and hip joint. These calculations were based on formulas presented by Mann \& Sprague (1980) and Simonsen et al. (1997). A net joint moment is the result of all moments of force acting about the actual joint. A moment pulling in a counter-clockwise direction is considered positive with the direction of movement from left to right. The sign of the moment does not necessarily indicate the direction of movement, and accordingly an extensor-dominated moment observed during flexion indicates an eccentric contraction of extensor muscles.

Estimates of instantaneous muscle-tendon lengths for the major muscles of the lower extremities were computed by vectorized algorithms based on anthropometric data reported by Frigo \& Pedotti (1978). The first derivative of these length changes was taken to yield shortening and lengthening velocities of the muscle-tendon units. Finally, these velocities were normalized to leg length and fiber lengths. The latter were based on a human cadaver study by Wickiewicz et al. (1983). The fiber lengths were: $\mathrm{m}$. vastus lateralis \& medialis (VL) 6.6 $\mathrm{cm}, \mathrm{m}$. rectus femoris (RF) $6.6 \mathrm{~cm}, \mathrm{~m}$. gastrocnemius medialis \& lateralis (GA) $5.1 \mathrm{~cm}, \mathrm{~m}$. gluteus maximus (GM) 14.0 $\mathrm{cm}, \mathrm{m}$. semitendinosus (ST) $15.8 \mathrm{~cm}, \mathrm{~m}$. semimembranosus (SM) $6.3 \mathrm{~cm}, \mathrm{~m}$. biceps femoris caput longum $(\mathrm{BFcl}) 8.5 \mathrm{~cm}$, $\mathrm{m}$. biceps femoris caput breve $(\mathrm{BFcb}) 13.9 \mathrm{~cm}$, m. soleus $(\mathrm{SO})$ $2.0 \mathrm{~cm}, \mathrm{~m}$. tibialis anterior (TA) $7.7 \mathrm{~cm}$.

The peak loading of the ACL was estimated with a model. When the peak net moment (extensor) about the knee joint occurred it was assumed that the patellar ligament would apply two force components to the tibia: one compressive component pulling in the longitudinal direction of the tibia and one component acting perpendicular to the tibia (shear force) pulling the bone in a forward direction. The shear force was then assumed to correspond to the load on the ACL. The size of the two force components was assumed to depend on the angle $(\beta)$ between the pulling direction of the patellar ligament and the longitudinal axis of the tibia (Fig. 1), which varies with the actual knee joint angle from approximately $+15^{\circ}$ near complete extension to approximately $-8^{\circ}$ at $90^{\circ}$ of flexion (Buff et al. 1988).

Table 1. Individual and mean values of peak knee joint moment, shear force (ACL loading) and knee joint angle during the side-cutting maneuver. The shear force and the knee joint angle correspond to the instant of peak knee joint moment

\begin{tabular}{lccc}
\hline Subject & $\begin{array}{c}\text { Peak knee joint } \\
\text { moment (Nm) }\end{array}$ & $\begin{array}{c}\text { Peak knee joint } \\
\text { shear force (N) }\end{array}$ & $\begin{array}{c}\text { Knee joint angle at } \\
\text { peak moment (deg) }\end{array}$ \\
\hline IR & 309 & 673 & 133 \\
KF & 99 & 215 & 116 \\
CR & 286 & 623 & 120 \\
GO & 213 & 464 & 142 \\
KJ & 278 & 606 & 127 \\
LP & 247 & 538 & 120 \\
Mean & 239 & 520 & 126 \\
SE & 31 & 68 & 4 \\
\hline
\end{tabular}

\section{Results}

Loading of the ACL

The net knee joint moment was extensor dominated during the whole contact phase in all cases (Fig. 2). The peak moment occurred about one-third into the contact phase and corresponded to the second peak of the vertical ground reaction force (Fig. 2). The contact phase could therefore be divided into a braking phase and a take-off phase, as also reported for vertical jumps (Gollhofer \& Kyrolainen 1991). In the following paragraphs data will be presented only for the braking phase (Fig. 2). The mean value of the knee joint angle was $126^{\circ}(116-142)\left(180^{\circ}\right.$ when fully extended) at the instant of the peak knee joint mo-
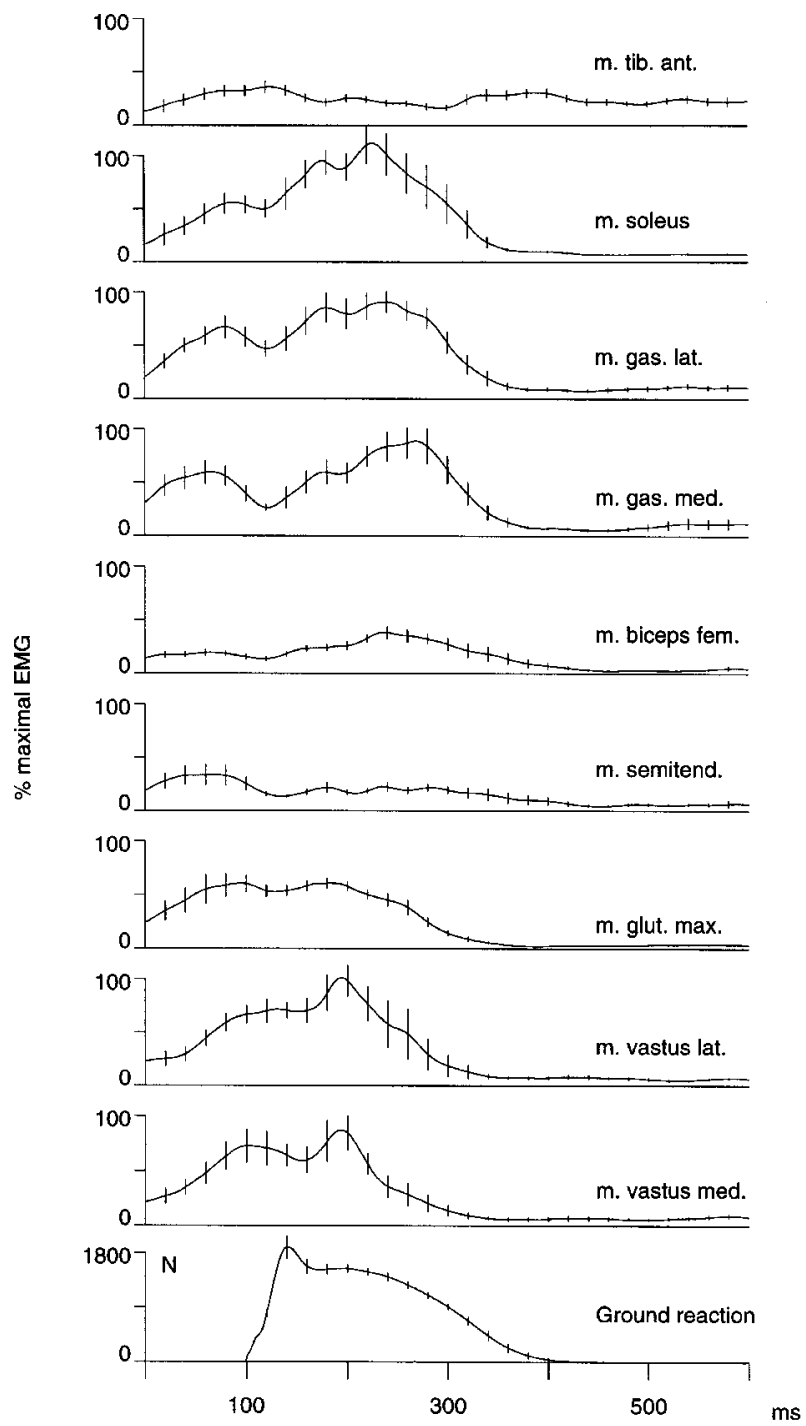

Fig. 3. Linear envelope EMGs from 9 muscles with the vertical ground reaction force at the bottom. The EMGs are averaged from 10 trials across 6 subjects and expressed relative to maximum EMG measured during isometric conditions. Vertical bars indicate standard error. 
Table 2. Average peak and mean EMG amplitudes for the braking phase with standard error (in parenthesis). All amplitudes are expressed relative to the maximal EMG amplitude measured during isometric conditions

\begin{tabular}{lccccccccc}
\hline & VM & VL & GM & SMT & BF & GAM & GAL & SO & TA \\
\hline Peak EMG & $110(26)$ & $129(16)$ & $90(18)$ & $27(5)$ & $37(7)$ & $75(15)$ & $101(11)$ & $120(16)$ & $42(6)$ \\
Peak EMG* & $87(18)$ & $101(17)$ & $61(5)$ & $34(10)$ & $39(6)$ & $89(16)$ & $91(10)$ & $112(20)$ & $36(6)$ \\
Mean EMG & $71(14)$ & $77(10)$ & $54(11)$ & $12(2)$ & $15(2)$ & $42(8)$ & $61(9)$ & $66(7)$ & $30(5)$ \\
\hline
\end{tabular}

* Denotes peak values averaged across 10 trials for each subject. The following muscles were recorded: $\mathrm{m}$. vastus medialis (VM), $\mathrm{m}$. vastus lateralis (VL), m. gluteus maximus (GM), m.m. semimembranosus et semitendinosus (SMT), m. biceps femoris (BF), m. gastrocnemius medialis (GAM), $m$. gastrocnemius lateralis (GAL), m. soleus (SO) and $\mathrm{m}$. tibialis anterior $(\mathrm{TA})$.

Table 3. Eccentric and concentric contraction velocities calculated during the braking phase of the side-cutting maneuver (average values across 6 subjects with standard error). The velocities are expressed in \% fiber length and \% leg length

\begin{tabular}{|c|c|c|c|c|c|}
\hline & VL & RF & GA & GM & SO \\
\hline$V_{\text {ecc }} \%$ fiber length/s & $216(10)$ & $253(35)$ & $255(78)$ & $34(8)$ & $973(208)$ \\
\hline \multirow[t]{2}{*}{$\mathrm{V}_{\text {ecc }} \%$ leg length/s } & $36(1)$ & $42(5)$ & $32(10)$ & $39(11)$ & $47(10)$ \\
\hline & ST & SM & $\mathrm{BFcl}$ & BFcb & TA \\
\hline$V_{\text {con }} \%$ fiber length/s & $222(22)$ & $427(41)$ & $251(36)$ & $100(9)$ & $194(40)$ \\
\hline$V_{\text {con }} \%$ leg length/s & $90(9)$ & $68(6)$ & $54(7)$ & $36(4)$ & 37 (8) \\
\hline
\end{tabular}

ment. The peak knee joint moment was $239 \mathrm{Nm}(99$ 309) (Table 1). These values were input to the knee joint model described above and resulted in a mean shear force/ACL-load of $520 \mathrm{~N}$ (215-673) (Table 1).

\section{EMG activity}

The temporal EMG activity of the nine recorded muscles is illustrated for all six subjects in Fig. 3. The vastus medialis $(\mathrm{VM})$ and lateralis $(\mathrm{VL})$ muscles were pre-activated prior to touch-down and stayed active until about $150 \mathrm{~ms}$ before toe off. The average across 10 trials and across subjects yielded peak EMG amplitudes during the braking phase of $87 \%$ for VM and $101 \%$ for VL (Table 2). When measured only during the single trial, which was analyzed by film, and then averaged for the six subjects, the peak amplitudes were slightly higher: $110 \%$ and $129 \%$, respectively (Table 2). The corresponding peak amplitudes for the semitendinosus/semimembranosus and biceps femoris muscles were $34 \%$ and $39 \%$ for 10 trials while $27 \%$ and $37 \%$ for the filmed trial, respectively (Table 2). For all muscles, the mean EMG amplitudes measured during the braking phase of the filmed trial showed the same pattern as the peak amplitudes (Table 2).

\section{Contraction velocity}

In Fig. 2 the variation in muscle-tendon length is superimposed on the EMGs for a typical trial. Inflection indicates lengthening while deflection indicates shortening. The mono-articular parts of the quadriceps muscle (VM and VL) were contracting eccentrically during the braking phase and concentrically during the take-off phase. In contrast, the bi-articular hamstring muscles ( $\mathrm{ST} / \mathrm{SM}$ and $\mathrm{BFcl}$ ) contracted concentrically during the whole braking phase (Fig. 2). The following lengthening and shortening velocities are mean values measured during the braking phase. The lengthening velocity of the vasti muscles was $216 \%$ fiber length/s (36\% leg length/s) and for the bi-articular rectus femoris $253 \%$ fiber length/s (42\% leg length/s) (Table 3). The shortening velocity for the semitendinosus muscle was $222 \%$ fiber length/s (90\% leg length/s) and for the semimembranosus $427 \%$ fiber length/s ( $68 \%$ leg length/s). For the long $(\mathrm{BFcl})$ and short $(\mathrm{BFcb})$ head of the biceps femoris muscle the shortening velocities were $251 \%$ fiber length/s (54\% leg length/s) and 100\% fiber length/s (36\% leg length/s) (Table 3). The gastrocnemius and the soleus muscles performed eccentric contractions at $255 \%$ fiber length/s (32\% leg length/s) and $973 \%$ fiber length/s (47\% leg length/s), respectively. The gluteus maximus muscle contracted eccentrically at 34\% fiber length/s (39\% leg length/s). Finally, the anterior tibial muscle contracted concentrically at 194\% fiber length/s (37\% leg length/s) (Table $3)$.

\section{Discussion}

The load of the ACL has not previously been investigated during a simulated side-cutting maneuver, which presumably places the ACL at risk of injury. Remarkably, the peak ACL loading in the present 
study was only $520 \mathrm{~N}$ on average, evaluated by inverse dynamics. Woo et al. reported the strength of the ACL at ultimate failure to be about $2000 \mathrm{~N}$ in specimens taken from young cadavers (Woo et al. 1991). The method used in the present study did not include rotational forces; however, it seems unlikely that rotation of the knee joint alone could ever increase the ACL loading 4-fold. The load on the ACL during a side-cutting maneuver is of interest with respect to understanding the injury mechanism of ACL injuries. The results of the present study suggest that a single side-cutting maneuver alone does not produce a load sufficient to create a clinical ACL injury. On the other hand, perhaps repeated loading of this magnitude is enough to produce material fatigue that will eventually result in complete disruption and clinical ACL injury. Since tendons and ligaments have a recovery period of about $10-12$ months in vivo (Noyes 1977), it is possible that total rupture of the ACL is preceded by numerous partial ruptures of the ligament over time.

In the present study it was noteworthy that the biarticular hamstring muscles were shortening during the braking phase and that they were only activated $27-37 \%$ of maximum, which suggest that their force production and ability to protect the ACL were marginal. However, it is difficult to determine the exact force-generating capacity due to shortening velocities of $222-427 \%$ fiber length/s. Vmax, the maximal shortening velocity measured during muscle contraction, and $V o$, the maximal shortening velocity for a hypothetical "unloaded" contraction, can be calculated using Hill's force-velocity relation. In various animal models, Vo has been reported to be 167-611\% fiber length/s. Grange et al. (1995) calculated Vo to be $469 \%$ fiber length/s in $\mathrm{m}$. extensor digitorum longus in the mouse. Asmussen \& Marechal (1989) calculated $V_{O}$ in $\mathrm{m}$. soleus to be $611 \%$ fiber length/s in the mouse, $167 \%$ fiber length/s in the guinea-pig and $416 \%$ fiber length in the rat. Vmax has been reported in a range of $482-1287 \%$ fiber length/s. In the cat, Spector et al. (1980) measured $V \max$ at the
Achilles tendon for the soleus muscle to be $482 \%$ fiber length/s while $1287 \%$ fiber length/s for the medial gastrocnemius muscle. Extrapolating from these animal data, the force production capacity of the hamstring muscles during the braking phase of the sidestep cutting maneuver should be reduced by approximately $30-100 \%$. Hansen (1961) measured the forcevelocity relation for the elbow flexor muscles in humans and expressed the shortening velocity in \% arm length/s. According to his data, the shortening velocities of the present study of $54-90 \%$ leg length/s of the bi-articular hamstring muscles would yield a force reduction of approx. $40 \%$. Therefore, the shortening velocity and the low activation level (EMG) of the hamstring muscles make it very unlikely that these muscles can effectively reduce the loading of the ACL during a side-cutting maneuver.

It has previously been speculated that increased cocontraction of the hamstring muscles during side-cutting could reduce the loading of the ACL (Colby et al. 1997). Furthermore, it has been hypothesized that strength training of the hamstring muscles could help with compensating for an ACL rupture or prevent damage to the intact ACL (Solomonow et al. 1989, Solomonow et al. 1987). According to the results of the present study, it seems unlikely that the side-cutting maneuver could be performed at all with an increased co-contraction of the hamstring group. The quadriceps femoris muscle was already activated close to maximum (Table 2), and therefore, if the hamstrings should increase their activity to produce a greater flexor moment about the knee joint, it is very unlikely that the desired movement pattern (the side-cutting maneuver) could be performed in an efficient way. Moreover, since the hamstring muscles were shortening rapidly during the movement, even a maximal activation of the muscle group would have a marginal effect due to the force-velocity relation.

Key words: ACL; hamstring muscles; force-velocity; EMG; side-cutting.

\section{References}

Andriacchi TP, Birac D. Functional testing in the anterior cruciate ligamentdeficient knee. Clin Orthop 1993: 40-7.

Asmussen G, Marechal G. Maximal shortening velocities, isomyosins and fibre types in soleus muscle of mice, rats and guinea-pigs. J Physiol (Lond) 1989: 416: 245-54.

Bencke J, Næsborg H, Simonsen EB, Klausen K. Motor pattern of the knee joint muscles during side-step cutting in European team handball. Influence on muscular coordination after an intervention study. Scand J Med Sci Sports 2000: 10: 68-77.

Buff HU, Jones LC, Hungerford DS. Experimental determination of forces transmitted through the patello-femoral joint. J Biomech 1988: 21: 17-23.

Colby S, Francisco A, Finch M, Beutler A, Garrett W. Electromyographic and kinematic analysis of cutting maneuvers: Implications for anterior cruciate ligament injury. American Society of Biomechanics 1997: (Abstract).
Draganich LF, Jaeger RJ, Kralj AR. Coactivation of the hamstrings and quadriceps during extension of the knee. J Bone Joint Surg Am 1989: 71: 1075-81.

Emerson RJ. Basketball knee injuries and the anterior cruciate ligament. [Review]. Clin Sports Med 1993: 12: 317-28.

Frigo C, Pedotti A. Determination of muscle length during locomotion. International Series on Biomechanics 1978: 2-A: 355-60.

Gollhofer A, Kyrolainen H. Neuro- 


\section{Simonsen et al.}

muscular control of the human leg extensor muscles in jump exercises under various stretch-load conditions. Int J Sports Med 1991: 12: 3440.

Grange RW, Cory CR, Van den Boom R, Houston ME. Myosin phosphorylation augments force-displacement and force-velocity relationships of mouse fast muscle. Am J Physiol 1995: 269: C713-24.

Hansen O. Thesis (in Danish). Copenhagen: August Krogh Institute, University of Copenhagen, 1961.

Jackson KM. Fitting of mathematical functions to biomechanical data. IEEE Trans Biomed Eng 1979: 26: 122-4.

Lindblad BE, Hoy K, Terkelsen CJ, Helleland HE. Handball injuries. An epidemiologic and socioeconomic study. Am J Sports Med 1992: 20: 4414.

Mann R, Sprague P. A kinetic analysis of the ground leg during sprint running. Res Q Exerc Sport 1980: 51: 334-48.

Myklebust T, Strand T, Engebretsen L, Nilsson S. Registation of anterior cruciate ligament injuries in the 3 upper divisions in Norwegian team handball: a prospective study. Scand J Med Sci Sports 1994: 3: 194 (Abstract).

Noyes FR. Functional properties of knee ligaments and alterations induced by immobilization. Clin Orthop 1977: 123: 210-42.

Simonsen EB, Dyhre-Poulsen P, Voigt M, Aagaard P, Fallentin N. Mechanisms contributing to different joint moments observed during human walking. Scand J Med Sci Sports 1997: 7: 1-13.

Solomonow M, Baratta R, Zhou BH, et al. The synergistic action of the anterior cruciate ligament and thigh muscles in maintaining joint stability. Am J Sports Med 1987: 15: 207-13.

Solomonow M, Baratta R, D'ambrosia $\mathrm{R}$. The role of the hamstrings in the rehabilitation of the anterior cruciate ligament-deficient knee in athletes. [Review]. Sports Med 1989: 7: 42-8. Spector SA, Gardiner PF, Zernicke RF, Roy RR, Edgerton VR. Muscle architecture and force-velocity characteristics of cat soleus and medial gastrocnemius: Implications for motor control. J Neurophysiol 1980: 44: 95160

Strand T, Tvedte R, Engebretsen L, Tegnander A. [Anterior cruciate ligament injuries in handball playing. Mechanisms and incidence of injuries]. [Norwegian]. Tidsskr Nor Laegeforen 1990: 110: 2222-5.

Strand T. Anterior cruciate ligament injuries in team handball. A prospective study of incidence rates and injury mechanism in the Bergen area. Scand $\mathbf{J}$ Med Sci Sports 1993: 3: 194.

Wickiewicz TL, Roy RR, Powell PL, Edgerton VR. Muscle architecture of the human lower limb. Clin Orthop 1983: 179: 275-83.

Winter DA. Biomechanics and motor control of human movement. 2nd edn. New York: John Wiley \& Sons, 1990.

Woo SL, Hollis JM, Adams DJ, Lyon RM, Takai S. Tensile properties of the human femur-anterior cruciate ligament-tibia complex. The effects of specimen age and orientation. Am J Sports Med 1991: 19: 217-25. 